\title{
Osteosarcoma: a comprehensive review
}

\author{
Amirhossein Misaghi ${ }^{1}$, Amanda Goldin ${ }^{1}$, Moayd Awad ${ }^{1,2}$, and Anna A Kulidjian ${ }^{1, *}$ \\ 1 Department of Orthopaedic Surgery, University of California San Diego, San Diego, CA, USA \\ 2 School of Medicine, University of Jeddah, Jeddah, Saudi Arabia
}

Received 20 January 2017, Accepted 15 April 2017, Published online 9 April 2018

\begin{abstract}
Osteosarcoma (OS) is a relatively rare tumor of bone with a worldwide incidence of 3.4 cases per million people per year. For most of the twentieth century, five-year survival rates for classic OS were very low. In the 1970s, the introduction of adjuvant chemotherapy in the treatment of OS increased survival rates dramatically. The current article reviews the various types of OS and analyzes the clinical and histological features. We also examine historical and current literature to present a succinct review of methods for diagnosis and staging, as well as treatment, and we also discuss some of the future directions of treatment.
\end{abstract}

Key words: Osteosarcoma, Review, Oncology, Sarcoma, Tumor.

\section{Introduction}

Osteosarcoma (OS) is a primary malignant bone tumor with a worldwide incidence of 3.4 per million people per year [1]. For most of the twentieth century, five-year survival rates for classic OS were $20 \%$. In the 1970 s, the introduction of adjuvant chemotherapy in the treatment of OS increased survival rates to $50 \%$ [2-4]. Before the mid-1970s, amputation was the routine treatment for high-grade OS. By 1990, the management of high-grade OS shifted to include more emphasis on chemotherapy and limb salvage. The current survival rate has increased to $>65 \%$ [5].

\section{Epidemiology}

OS is a rare sarcoma that has the histological findings of osteoid production in association with malignant mesenchymal cells [6]. OS is the third most common cancer in adolescence, with only lymphomas and brain tumors being more prevalent, and with an annual incidence of 5.6 cases per million children under the age of 15 [7-9]. Peak incidence is in the second decade of life [10,11]. Before the age of five, OS is rare [12]. OS arises sporadically, with few cases associated with known inherited defects in cell cycle regulation, but about $70 \%$ of tumor specimens demonstrating a chromosomal abnormality. These commonly involve mutations in tumor-suppressor genes or in DNA helicases [13].

\footnotetext{
*Corresponding author: akulidjian@gmail.com
}

\section{Types}

The World Health Organization's histologic classification of bone tumors divides OS into central, intramedullary, and surface tumors, with a number of subtypes under each group [14].

\section{Central}

\section{Conventional osteosarcoma}

Conventional OS is the most common type of OS and represents $80 \%$ of all osteosarcoma cases primarily affecting individuals in the first and second decades of life. It can be subdivided into osteoblastic, chondroblastic, and fibroblastic groups depending on the predominant features of the cells; there are no significant differences in clinical outcomes among these categories [14]. OS is typically high grade and originates in the intramedullary cavity. On radiographs OS can be osteolytic or osteoblastic, or both. Eighty percent of cases are located in the metaphysis of long bones, but OS can also arise in the diaphysis of long bones as well as the axial skeleton [15]. On histology, evidence of bone or osteoid production by the tumor cells is a requirement for diagnosis [16].

\section{Telangiectatic osteosarcoma}

Telangiectatic osteosarcoma (TOS) accounts for $4 \%$ of OS [17]. Histologically, dilated blood-filled cavities and high-grade sarcomatous cells on the septae and peripheral rim characterize 
TOS. Radiographically, TOS is metaphyseal, with geographic patterns of bone destruction and a wide zone of transition. Moth-eaten or permeative destruction can be seen [18]. It is important to differentiate TOS from aneurysmal bone cysts (ABC) on imaging. $\mathrm{ABC}$ is described as an eccentric lytic lesion with a "blown-out" space in the bone. The two lesions are known to appear radiographically similar and cases of mistaken TOS for ABC have been reported [19-21]. Although it was believed that the prognosis of TOS is worse than the Conventional Type, recent studies suggest that there is no difference between the two types [22, 23].

\section{Small-cell osteosarcoma}

Small-cell osteosarcoma (SOS) constitutes 1-2\% of all OS. The histological features of SOS show cells that are small, and have round hypochromatic nuclei with little nuclear polymorphism, similar to Ewing's sarcoma [24, 25]. However, while the production of osteoid by tumor cells confirms the diagnosis for OS, it is not a characteristic feature of Ewing's sarcoma [26]. A destructive process with lytic areas and sclerosis is found on radiographs [25].

\section{Low-grade osteosarcoma}

Low-grade osteosarcoma (LOS) accounts for $1-2 \%$ of all OS. However, LOS generally affects persons in the third or fourth decade of life [17, 27]. LOS can be difficult to recognize, as it is low grade and may resemble parosteal osteosarcoma, fibrous dysplasia, or desmoplastic fibroma [28]. While there is a risk of transformation to conventional OS if treated with curettage alone, the prognosis is significantly better in LOS [29].

\section{Surface}

\section{Parosteal osteosarcoma}

Parosteal osteosarcoma (PAOS) is a low-grade osteosarcoma that originates from the periosteum. PAOS represents $4-6 \%$ of OS and commonly affects the posterior aspect of the distal femur. It may also occur in other sites including the proximal humerus and proximal tibia [30, 31]. Radiographs demonstrate a densely ossified and lobulated mass, while the medullary cavities are spared [32]. Histologically, PAOS exhibits streams of bone trabeculae that show a high degree of parallel orientation, similar to what may be seen in a periosteal new bone reaction [26].

\section{Periosteal osteosarcoma}

Periosteal osteosarcoma (PIOS) has a matrix component that is mainly cartilaginous and less common than parosteal. PIOS tends to arise between the cortex and the cambium layer of the periosteum, and therefore a periosteal reaction is usually visible on radiographs [33]. On histopathologic examination, an intermediate-grade tumor is seen, containing a cartilaginous matrix with areas of calcification [26].

\section{High-grade surface osteosarcoma}

High-grade surface osteosarcoma (HGSOS) constitutes less than $1 \%$ of all OS [17] and manifests as a surface lesion with a high-grade appearance histologically [34]. Local growth is accelerated in HGSOS more than in parosteal osteosarcoma. HGSOS has the same malignant potential as the conventional type, therefore some degree of localized invasion of the cortex and endosteum may be seen [26]. Radiographically, HGSOS demonstrates a surface lesion with partial mineralization, and the tumor may extend to surrounding soft tissues [35].

\section{Diagnosis and staging}

The technology and techniques used to diagnose osteosarcoma have improved over the past several decades [36]. For any suspected bone lesion, a preoperative imaging protocol should be followed, which includes taking at least two X-ray views of the whole bone and the adjacent joint. Radiographs will show an ill-defined lesion arising at the metaphysis of bone, with osteoblastic and/or osteolytic areas, periosteal reaction, and a soft tissue mass [37-40].

Magnetic resonance imaging (MRI) is warranted to evaluate the lesion's invasion into the soft tissue and neurovascular structures, level of bone marrow replacement, skip lesions, and extension into the bordering joint [37-41]. In 1994, Schima et al. investigated the usefulness of contrast-enhanced MRI in determining intra-articular tumor extension in patients with OS. Ten of 46 patients had extension of tumor into the joint on pathology, of which all were preoperatively identified on MRI. Eleven other tumors showed potential intra-articular extension on MRI, providing a sensitivity of $100 \%$ and a specificity of $69 \%$. Pathologically, 12 patients were found to have transphyseal involvement, of whom all had preoperative evidence of transphyseal involvement on MRI, providing a sensitivity and specificity of $100 \%$. The authors concluded that T1-weighted contrast-enhanced imaging is useful for detecting intra-articular OS involvement [39]. In 1991, Shuman et al. showed that fat-suppression MRI allowed exposure of a greater quantity of abnormal tissue than proton density and T2-weighted imaging; yet, they suggested that fat suppression may cause fat planes to appear ambiguous, which can cause difficulty in precise staging of disease. They did not use contrast-enhanced T1-weighted images in this study [42].

Computed tomography (CT) scans are useful in defining cortical irregularities, fracture sites, mineralization, and neurovascular involvement [37-40]. Bone scintigraphy can help show polyostotic involvement, metastases, and intraosseous tumor extension. Angiography may aid in showing vascular anatomy. This is helpful for preoperative planning in patients with tumors at the proximal tibia or shoulder girdle, as these are areas with common vascular anatomic anomalies $[39,40]$. Positron emission tomography (PET) scans can be used to assess the primary lesion(s) and to detect metastatic lesions in other bones and the lungs [37]. Some suggest using PET scans to assess the histologic response of the disease to chemotherapy as well as to predict progression-free survival [40]. CT and/or 
X-ray of the chest should be completed to assess pulmonary metastases, as this is the most common location of metastatic disease in osteosarcoma [36, 37, 39, 40, 43]. It is reasonable to obtain a repeat CT scan 6-12 weeks following the first, as it is difficult to detect metastatic lesions that are less than $5 \mathrm{~mm}$ in size [40].

A biopsy is essential in the diagnosis of OS. Eventual tumor resection must include the biopsy tract, as this tract could get contaminated with tumor cells. The surgeon should choose a biopsy tract that will be included in this future surgery [38]. Preferably, the surgeon performing the biopsy would be the same surgeon to ultimately perform the resection; if this is not possible, an experienced radiologist may perform the biopsy with imaging assistance, or an experienced surgeon may perform a percutaneous biopsy without radiologic guidance [40, 44]. Open biopsy was once considered the gold standard due to its accuracy rate of $98 \%$; however, there are associated complications with open biopsy, especially if the biopsy is conducted outside of the ultimate treatment facility [45]. Core biopsy is preferred because there is less risk of local contamination. This is important in patients who may have limb-sparing surgery [37, 39]. Hau et al. set to determine whether CT-guided biopsy is as accurate as open biopsy in diagnosing musculoskeletal tumors. They retrospectively reviewed 359 patients who had undergone CT-guided biopsy and found an overall accuracy of $71 \%$; of these, 258 were CT-guided core biopsies and had an accuracy of $74 \%$, while 101 were fine needle aspirations and had an accuracy of $63 \%$ [45]. They concluded that while both core needle and open biopsy provide adequate tissue for accurate analysis, fine needle aspiration should not be used, as it does not provide a large enough sample for a precise diagnosis [40, 45]. Welker et al. conducted a retrospective review of 173 core needle biopsy procedures, of which 90 were performed without radiologic guidance, and found that $88.2 \%$ of these biopsies were sufficient for diagnosis. In this study, percutaneous needle biopsy provided a positive predictive value of $100 \%$, a negative predictive value of $82 \%$, a sensitivity of $81.8 \%$, and a specificity of $100 \%$. They deemed percutaneous needle biopsy a safe and effective method for diagnosing musculoskeletal masses [44]. Similarly, the prospective study by Skrzynski et al. found an accuracy rate of $84 \%$ for patients undergoing a closed needle biopsy and found this to be an accurate and less expensive procedure [46].

After the biopsy is performed, a frozen section will sometimes be completed. Histologically, OS will appear as osteoblastic, chondroblastic, or fibroblastic [16, 47]. Many tumors will display aspects of all three cell types and matrix [16], and findings should be reviewed by a pathologist with experience in musculoskeletal pathology [39]. Mitsuyoshi et al. reviewed the biopsies of 157 patients and found that an experienced musculoskeletal pathologist was able to distinguish malignant from benign lesions with $100 \%$ accuracy in bone tumors, and obtain a specific diagnosis in $96 \%$ of the cases of bone tumors [48].

There is no laboratory test that is diagnostic for OS; however, complete blood count, basic metabolic panel, renal and liver function tests, and urinalysis are all useful to assess the patient's baseline organ function prior to the start of chemotherapy. Osteoblastic activity can be assessed with alkaline phosphatase levels, and lactate dehydrogenase levels can be used to assess osteoclastic activity [38]. Recent studies have demonstrated that $\mathrm{C}$-reactive protein (CRP) has prognostic value in OS, as patients with higher levels of CRP have a statistically higher probability of death due to disease. Funovics et al. demonstrated that patients whose preoperative CRP was greater than $1.0 \mathrm{mg} / \mathrm{dL}$ had a significantly worse prognosis than those with CRP levels below $0.02 \mathrm{mg} / \mathrm{dL}[49,50]$.

The staging classification used in osteosarcoma is the Musculoskeletal Tumor Society staging scheme, also known as the Enneking system. This system establishes whether a tumor is low or high grade (I or II), whether the tumor is intraor extra-compartmental (A or B), and whether any metastases are present (III). Stage IA represents a low-grade tumor that is intra-cortical, IB represents a low-grade tumor that is extra-cortical, IIA represents a high-grade tumor that is intracortical, and IIB represents a high-grade tumor that is extracortical. Metastatic disease automatically places the patient in the stage III category [38, 40, 41]. Most commonly, OS patients are diagnosed at stage IIB [38].

\section{Treatment}

Conventional treatment for OS consists of a combination of neoadjuvant and adjuvant chemotherapy, and surgery $[4,51]$. Prior to the use of chemotherapy, there was less than a $20 \%$ survival rate in high-grade conventional osteosarcoma even with surgical amputation, indicating the presence of micrometastases (typically pulmonary) prior to surgery $[4,52]$. The low grade can typically be treated with excision alone and chemotherapy is avoided if final pathology confirms low grade.

\section{Surgical treatment}

The goal of tumor surgery is complete resection of disease via wide excision of the tumor [53]. Surgical options can be divided into limb salvage versus amputation.

\section{Limb salvage}

Limb salvage surgical techniques provide a safe methodology of treatment for $85-90 \%$ of patients with OS [54-56]. There are two essential steps of limb salvage, including resection and reconstruction. Resection is crucial to the elimination of disease. It should include excision of previous biopsy sites and tracts with at least a $2 \mathrm{~cm}$ margin. All major vessels should be identified prior to ligation. Preoperative imaging, such as bone and CT scan, should be utilized to determine the necessary quantity of bone to be osteotomized. This should be around $6-7 \mathrm{~cm}$ distal to the lesion to ensure clear margins $[57,58]$. Custom jigs generated by computers have also gained favor as a tool to improve accuracy in wide resection of OS [59-61]. Khan et al. conducted a study on six pairs of matched cadaver femurs utilizing computer-aided design software for one of the femurs and manual resection for the other. They found a significantly higher deviation from the preoperative 
plan in their manual femurs compared to the ones with custom jigs [61]. Computer-aided navigation can be especially useful in pelvic and sacral tumors, as it allows safe margins to be obtained as planned preoperatively, without over-resection of weight-stabilizing bone [59, 60]. Tumor resection surgery in patients who are skeletally immature brings up the issue of physeal destruction and the possibility of growth disturbances. Traditionally, location of the tumor through the growth plate was a contraindication for limb salvage and an indication to amputate. Now, current treatments include resection with expandable growth endoprosthesis, allograft endoprosthetic composites, or rotationplasty [62]. Resections around the joints are challenging, and joint contamination precludes limb preservation surgery, necessitating an amputation. Some centers advocate preservation of the joint through resections through the growth plate [53, 63]. In 1994, Canadell described a method of combining distraction osteogenesis with an external fixator with tumor resection in order to try and decrease growth discrepancies. They operated on 20 patients, of whom none had local recurrences and three had pulmonary metastases. They ultimately determined this method a safe and effective way to resect tumor but maintaining the epiphysis of long bones [63]. Other centers have simulated these results [53], however experience is limited.

Reconstruction is the next step in limb salvage. It should be noted that non-weight-bearing bones, such as the clavicle or proximal fibula, do not require reconstruction, as excision alone does not cause functional deficits [58, 64]. When reconstruction is utilized in weight-bearing bones, it can be divided into endoprosthetic replacement and biological reconstruction. Endoprosthetic replacement is a form of limb salvage reconstruction, and has been reported to have good functional outcomes and better cosmetic and psychological benefits in comparison to other forms of treatment, including amputation and rotationplasty [65]. The design of these implants includes modular, custom-made, and growing implants for the skeletally immature. Since the 1990s implant design has been modular with titanium segments and cobalt-chrome alloy tapers in order to prevent cold welding [66]. Titanium alloys are associated with a lower rate of late infections than cobalt-chrome alloys. Silver-coated titanium megaprostheses are thought to reduce infection rate further [67]. Iodine coating of titanium implants has also been shown to decrease infection risk [68]. The first endoprostheses were custom designed. Modular prostheses allow for use of off-the-shelf components that are less expensive and timely to make than custom-made, and have proven to have good survivorship [66, 69]. Ahlmann et al. retrospectively reviewed 211 patients who had undergone limb salvage with modular endoprosthesis, and found a survivorship rate of $78 \%$ at five years post op and $60 \%$ at 15 years post op, which generally outlasted the survival rates of the patients [69]. Schwartz et al. compared the survivorship of 85 patients with modular implants and 101 patients with custom-designed implants and found that there was 15-year survivorship of $93.7 \%$ and $51.7 \%$, respectively [66]. Finally, in the case of children, expandable prosthesis can be utilized, which involves a prosthesis that allows for interval lengthenings via a series of minor surgical procedures. The growth plates of the affected bone are removed, and the prosthesis is lengthened by $1-2 \mathrm{~cm}$ per surgery, in order to correlate with the contralateral, healthy extremity [62].

Biologic replacement is the second form of limb reconstruction, which includes allograft, autograft, recycled autografts, and allograft prosthetic composite reconstructions. Massive bone allografts have been in use since as early as 1908, however debate regarding their effectiveness and durability continues. Donati et al. performed a retrospective review evaluating 92 patients who had undergone massive allograft reconstruction. Forty-five percent and $29 \%$ had an "excellent" and "good" outcome, respectively, while $15 \%$ of the allografts failed [70]. Similarly, Gebhardt et al. examined a cohort of 53 patients with allograft reconstruction, and of the 38 who did not have a recurrence of the disease there was a $70 \%$ satisfactory rate [71]. These authors concluded that overall bone allografts can be an effective method of tumor reconstruction after resection [70, 71]. Finally, allograft prosthetic composites (APC) combine implants with allograft for reconstruction. APC arthroplasty is utilized for weight-bearing joints, including the hip and knee. It combines the benefits of a biologic graft, including better reinsertion of soft tissues and preservation of anatomy, with the stability and ability to immediately weight bear of a prosthesis. It is at risk for nonunion and infection $[70,72]$.

Autografts can be used in a number of ways. The fibula is an ideal bone for autograft harvest, as it is long, tubular, relatively superficial, and minimally load sharing. It can be vascularized or not, however non-vascularized graft is dependent on the blood supply and bone quality it is placed into. Vascularized fibula tends to have a reduced time to union and faster hypertrophy than non-vascularized [73]. The autogenous recycling method involves tumor-bearing autografts being heated, frozen, or irradiated. Benefits of pasteurized autografts include preserving anatomy and bone-inductive activity, but complications include bone absorption, fracture, pseudoarthrosis, and infection $[68,74]$. Tsuchiya et al. treated 33 malignant bone tumors with pedicle frozen autograft (by liquid nitrogen), and obtained excellent results in $75.7 \%$ of patients, but also had complications in $48 \%$, including infection, fracture, and recurrence [68].

Some studies have reported slightly higher recurrence rates with limb salvaging compared to amputation, however the overall survival rate of patients who recur is comparable [75]. In fact, some studies show that survival rates are higher with limb salvage than amputation. In 2001, Ferrari et al. demonstrated an eight-year-survival rate of 62 in patients undergoing limb salvage, compared to $43 \%$ for those undergoing amputation [55]. Endoprosthetic replacement in tumor surgery has been shown to lead to improved quality of life. Lang et al. did a retrospective review to analyze the sporting abilities in 27 patients with OS who received limb-salvaging modular endoprostheses. They found that by five years postoperation, the same percentage of patients who played sports previously played sports post-operatively. They concluded that patients can reach high levels of sports after a modular endoprosthesis, and the potential to do so depends more on preoperative activity rather than the procedure and implant itself [76]. Many now consider limb salvage to be the preferred treatment for malignant sarcomas [77]. 


\section{Amputation}

Amputation, once the standard surgical treatment of OS, is now typically reserved to the non-resectable tumor with soft tissue and neuromuscular contamination not amenable to repair. Many studies argue that limb salvaging surgery provides better daily function than amputation and is equal, if not better, in terms of survival [62, 77-79]. A novel surgical treatment includes osteointegration implants, which are used as an adjunct to treatment in amputees to increase function. Branemark et al. conducted a prospective study of 51 patients who had undergone transfemoral amputations, either due to tumor or trauma. These patients had a survival rate of $92 \%$ at two years, and overall reported increased use of prosthetic and quality of life [80].

Rotationplasty involves resection of the distal femur, followed by rotation of the lower leg $180^{\circ}$ thus turning the ankle joint into a "knee" joint [78, 81, 82], with the gastrocnemius and soleus plantar flexors becoming "knee" extensors [81]. It was first described in 1930 by Borggreve for a shortened leg after a patient had tuberculous ankylosis of the knee joint; however, it was not until 1974 that the procedure was described as a treatment for OS [78, 81]. Rotationplasty has shown good functional and rehabilitative results, especially in children and active adults [78, 81, 82]. However, its odd appearance causes psychological problems in some patients $[65,83]$.

Salzer et al. [78] conducted a study in 1981 with 15 patients who underwent rotationplasty for OS. Patients ranged from six to 32 years old and were followed from six to 63 months post-operatively. Three patients died of metastases at 24 and 25 months post-operatively, while 12 patients had no evidence of disease at the completion of the study. Functionally, all patients could fully extend their "knees" and achieved flexion between $70^{\circ}$ and $90^{\circ}$. In 2015, Gradl et al. aimed to describe the long-term quality of life results of rotationplasty and in this study the patients overall had positive experiences after their rotationplasties [83].

\section{Chemotherapy treatment}

Prior to the 1970s, chemotherapy was not used for osteosarcoma and survival rates were dismal. In 1972, MD Anderson released a study treating their osteosarcoma patients with chemotherapy and presenting a two-year survival rate of $50 \%$. In 1981, a prospective trial began that compared the outcomes of 27 patients treated without any adjuvant chemotherapy to 32 patients receiving either Adriamycin, high-dose methotrexate, or a combination of bleomycin, Cytoxan, and actinomycin-D. In 1984, the trial was discontinued when it became clear that the patients receiving chemotherapy had a statistically significant advantage, as $55 \%$ remained disease-free at two years, in contrast to only $20 \%$ of the non-chemotherapy group disease-free. Survival at two years was also significant, with $80 \%$ versus $48 \%$ of the patients remaining alive in the treatment and control groups, respectively [4].

The standard of care for osteosarcoma is currently neoadjuvant chemotherapy, surgery, and adjuvant chemotherapy.
The four chemotherapy agents that are in nearly all treatment regimens include methotrexate with leucovorin rescue, doxorubicin, cisplatin, and ifosfamide. Patients who have metastatic disease may also be treated with etoposide. Tumor necrosis response to neoadjuvant chemotherapy dictates the overall response to treatment. O'Kane et al. retrospectively reviewed 97 patients with osteosarcoma treated with chemotherapy and surgery, and found that those who had greater than $90 \%$ tumor necrosis had an $82 \%$ five-year survival rate, while those who had less than $90 \%$ tumor necrosis had a $68 \%$ five-year survival rate [52]. The idea of "dose intensity" is important as well. Imran et al. retrospectively reviewed 703 charts of patients with localized OS, and found that those who waited over 21 days after surgery to resume chemotherapy had a significantly higher mortality rate. The authors suggest restarting chemotherapy within the first 21 days postoperatively in order to maintain dose intensity [84].

\section{Radiation treatment}

Radiation treatment has a controversial role in the treatment of OS due to its questionable effectiveness and associated risk of infection. An interesting application as an adjunct to low-cost reconstructive modality has been popularized in Japan. A 2013 study retroactively reviewed 101 patients with sarcoma (37 of whom had OS) after receiving extracorporeal irradiation (ECI) and yielded some promising results. The ECI consists of en bloc resection of the involved bone, treatment of each bone segment with 50 Gy radiation, and ultimate replantation of the bone. None of the 37 patients with OS had disease recurrence. The authors promote ECI as a low-cost treatment that is effective at preventing disease recurrence and carries a low risk of infection [51].

\section{Future directions}

There have been no significant advances in the treatment of the disease over the last 10 plus years, however advancements are slowly being made in the treatment of OS as more is being understood about the pathophysiology of the disease. Novel drug delivery systems and immunotherapies are being developed, and old dogmas of neoadjuvant chemotherapy and surgical resection are being challenged. Jones et al. evaluated 24 patients with distal femoral OS who underwent MRI pre- and post-neoadjuvant chemotherapy, and determined that although neoadjuvant chemotherapy did affect surgeon planning of resection level, it did not do so in a reliable direction. With MRI's obtained post-neoadjuvant chemotherapy, more surgeons elected to proceed with amputation, implying that neoadjuvant chemotherapy does not offer significant clinical benefit in the anatomical planning of resection level. A proposed theory as to the dogma of chemotherapy was noting that edema improves post-treatment, leading to the belief that chemotherapy improves resectability [85].

New reconstructive modalities are also being proposed by Tsuchiya et al. They have studied the long-term outcomes of utilizing liquid nitrogen (LN2) to kill tumor cells and replanting patient's own tissue after LN2 treatment. 
They report on 17 osteosarcomas treated with a pedicle frozen autograft, with 14 cured of the disease, two local recurrences, and one leading to distal metastasis [68]. This treatment may offer a cost-effective perfectly matched autograft as an alternative to allografts and endoprosthesis, though long-term effectiveness needs to be further studied.

Advances in treatment are also being made on the molecular level. Mason et al. made promising strides in a canine model of OS, using an attenuated listeria vaccine to deliver and induce innate HER2/neu immunity, leading to a significant reduction in metastatic disease and increases in survival [86].

Tyrosine kinase inhibitors have been investigated, and in vitro studies have shown that they reduce motility, colony formation, and invasiveness of disease, and may be beneficial in managing cases of OS metastases [87].

\section{Summary}

Osteosarcoma is a rare bone tumor found in areas of rapid bone turnover, most commonly the distal femur and proximal tibia of adolescent patients. Early on, treatment of osteosarcoma typically involved surgical resection in the form of amputation or reconstruction with auto- or allograft. With the addition of neoadjuvant chemotherapy to treatment protocols, five-year survival rates have dramatically increased. The current treatment of osteosarcoma involves neoadjuvant chemotherapy, wide resection, followed by adjuvant chemotherapy, with strict emphasis on the overall intensity of treatment and resumption of the post-resection chemotherapy as soon as possible. Progress is being made in the areas of immune therapy and targeted chemotherapy, and the investigation of newer treatment strategies has yielded promising early results.

\section{References}

1. Mirabello L, Troisi RJ, Savage SA (2009) International osteosarcoma incidence patterns in children and adolescents, middle ages and elderly persons. Int J Cancer 125, 229-234.

2. Harvei S, Solheim Ø (1981) The prognosis in osteosarcoma: Norwegian national data. Cancer 48, 1719-1723.

3. Sutow WW, Sullivan MP, Fernbach DJ, Cangir A, George SL (1975) Adjuvant chemotherapy in primary treatment of osteogenic sarcoma. A Southwest Oncology Group study. Cancer 36, 1598-1602.

4. Eilber ByF, Giuliano A, Eckardt J, Patterson K, Moseley S, Goodnight J (1987) Adjuvant chemotherapy for osteosarcoma: a randomized prospective trial. J Clin Oncol 5, 21-26.

5. Simon MA, Aschilman MA, Thomas N, Mankin HJ (1986) Limb-salvage treatment versus amputation for osteosarcoma of the distal end of the femur. J Bone Joint Surg Am 68, 1331-1337.

6. Raymond AK, Jaffe N (2009) Osteosarcoma multidisciplinary approach to the management from the pathologist's perspective, in Pediatric and Adolescent Osteosarcoma. p. 63-84.

7. Abate ME, Longhi A, Galletti S, Ferrari S, Bacci G (2010) Non-metastatic osteosarcoma of the extremities in children aged 5 years or younger. Pediatr Blood Cancer 55, 652-654.
8. Kager L, Zoubek A, Potschger U, Kastner U, Flege S, Kempf-Bielack B, Branscheid D, et al. (2003) Primary metastatic osteosarcoma: presentation and outcome of patients treated on neoadjuvant cooperative osteosarcoma study group protocols. J Clin Oncol 21, 2011-2018.

9. Pakos EE, Nearchou AD, Grimer RJ, Koumoullis HD, Abudu A, Bramer JAM, Jeys LM, et al. (2009) Prognostic factors and outcomes for osteosarcoma: an international collaboration. Eur J Cancer 45, 2367-2375.

10. Cho WH, Song WS, Jeon DG, Kong CB, Kim MS, Lee JA, Yoo JY, Kim JD, Lee SY (2010) Differential presentations, clinical courses, and survivals of osteosarcomas of the proximal humerus over other extremity locations. Ann Surg Oncol 17, 702-708.

11. Kaste SueC, Liu T, Billups CA, Daw NC, Pratt CB, Meyer WH (2004) Tumor size as a predictor of outcome in pediatric nonmetastatic osteosarcoma of the extremity. Pediatr Blood Cancer 43, 723-728.

12. Kager L, Zoubek A, Dominkus M, Lang S, Bodmer N, Jundt G, Klingebiel T, Jürgens H, Gadner H, Bielack S (2010) Osteosarcoma in very young children. Cancer 116, 5316-5324.

13. Hayden JB, Hoang BH (2006) Osteosarcoma: basic science and clinical implications. Orthop Clin North Am 37, 1-7.

14. Ozaki T, Flege S, Liljenqvist U, Hillmann A, Delling G, Salzer-Kuntschik M, Jürgens H, Kotz R, Winkelmann W, Bielack SS (2002) Osteosarcoma of the spine: experience of the cooperative osteosarcoma study group. Cancer 94, 1069-1077.

15. Bielack SS, Kempf-Bielack B, Delling G, Exner GU, Flege S, Helmke K, Kotz R, et al. (2002) Prognostic factors in highgrade osteosarcoma of the extremities or trunk: an analysis of 1,702 patients treated on neoadjuvant cooperative osteosarcoma study group protocols. J Clin Oncol 20, 776-790.

16. Klein MJ, Siegal GP (2006) Osteosarcoma anatomic and histologic variants. Am J Clin Pathol 125, 555-581.

17. Fletcher CD, Krishnan Unni K, Mertens F (2002) Pathology and genetics of tumours of soft tissue and bone. IARC 4 .

18. Murphey MD, wan Jaovisidha S, Temple HT, Gannon FH, Jelinek JS, Malawer MM (2003) Telangiectatic osteosarcoma: radiologic-pathologic comparison. Radiology 229, 545-553.

19. Kaufman RA, Towbin RB (1981) Telangiectatic osteosarcoma simulating the appearance of an aneurysmal bone cyst. Pediatr Radiol 11, 102-104.

20. Kransdorf MJ, Sweet DE (1995) Aneurysmal bone cyst: concept, controversy, clinical presentation, and imaging. AJR Am J Roentgenol 164, 573-580.

21. Mervak TR, Krishnan Unni K, DJ Pritchard, RA Mcleod (1990) Telangiectatic osteosarcoma. Clin Orthop Relat Res 270, 135-139.

22. Weiss A, Khoury JD, Hoffer FA, Jianrong Wu, Billups CA, Heck RK, Quintana Juan, Poe Debbie, Rao BN, Daw NC (2007) Telangiectatic osteosarcoma: the St. Jude Children's Research Hospital's experience. Cancer 109, 1627-1637.

23. Bacci G, Ferrari S, Ruggieri P, Biagini R, Fabbri N, Campanacci L, Bacchini P, Longhi A, Forni C, Bertoni F (2001) Telangiectatic osteosarcoma of the extremity: neoadjuvant chemotherapy in 24 cases. Acta Orthop Scand 72, 167-172.

24. Sim FH, Unni KK, Beabout JW, Dahlin DC (1979) Osteosarcoma with small cells simulating Ewing's tumor. J Bone Joint Surg Am 61, 207-215.

25. Nakajima H, Sim FH, Bond JR, Unni KK (1997) Small cell osteosarcoma of bone. Review of 72 cases. Cancer 79, 2095-2106. 
26. Klein MJ, Siegal GP (2006) Osteosarcoma: anatomic and histologic variants. Am J Clin Pathol 125, 555-581.

27. Andresen KJ, Sundaram M, Unni KK, Sim FH (2004) Imaging features of low-grade central osteosarcoma of the long bones and pelvis. Skeletal Radiol 33, 373-379.

28. Bertoni F, Bacchini P, Fabbri N, Mercuri M, Picci P, Ruggieri P, Campanacci M (1993) Osteosarcoma. Low-grade intraosseoustype osteosarcoma, histologically resembling parosteal osteosarcoma, fibrous dysplasia, and desmoplastic fibroma Cancer 71, 338-345.

29. Unni KK, Dahlin DC, McLeod RA, Pritchard DJ (1977) Intraosseous well-differentiated osteosarcoma. Cancer 40, 1337-1347.

30. Johnson K, Davies AM, Mangham DC, Grimer RJ (1999) Parosteal osteosarcoma of a metatarsal with intramedullary invasion. Skeletal Radiol 28, 111-115.

31. Hewitt KM, Ellis G, Wiggins R, Bentz BG (2008) Parosteal osteosarcoma: case report and review of the literature. Head Neck 30, 122-126.

32. Okada K, Frassica FJ, Sim FH, Beabout JW, Bond JR, Unni KK (1994) Parosteal osteosarcoma. A clinicopathological study. J Bone Joint Surg Am 76, 366-378.

33. Unni KK, Dahlin DC, Beabout JW (1976) Periosteal osteogenic sarcoma. Cancer 37, 2476-2485.

34. Wold LE, Unni KK, Beabout JW, Pritchard DJ (1984) High-grade surface osteosarcomas. Am J Surg Pathol 8, 181-186.

35. Okada K, Krishnan Unni K, Swee RG, Sim FH (1999) High grade surface osteosarcoma. Cancer 85, 1044-1054.

36. Mialou V, Philip T, Kalifa C, Perol D, Gentet JC, Marec-berard P, Pacquement Helene, Chastagner P, Defaschelles AS, Hartmann O (2005) Metastatic osteosarcoma at diagnosis prognostic factors and long-term outcome - the French pediatric experience. Cancer 104, 1100-1109.

37. Majó J, Cubedo R, Pardo N (2010) Treatment of osteosarcoma. A review. Rev Esp Cir Ortop Traumatol 54, 329-336.

38. Messerschmitt PJ, Garcia RM, Abdul-Karim FW, Greenfield EM, Getty PJ (2009) Osteosarcoma. J Am Acad Orthop Surg $17,515-527$.

39. Aboulafia AJ, Malawer MM (1993) Surgical management of pelvic and extremity osteosarcoma. Cancer 71, 3358-3366.

40. Geller DS, Gorlick R (2010) Osteosarcoma: a review of diagnosis, management, and treatment strategies. Clin Adv Hematol Oncol 8, 705-718.

41. Schima W, Amann G, Stiglbauer R, Windhager R, Kramer J, Nicolakis M, Farres MT, Imhof H (1994) Preoperative osteosarcoma: imaging staging of efficacy of MR in detecting joint involvement. Am J Roentgenol 163, 1171-1175.

42. Shuman WP, Patton AM, Baron AL, Uddell AM, Conrad EU, Richarson ML (1991) Comparison of STIR and spin-echo MA imaging at $1.5 \mathrm{~T}$ in 45 suspected extremity tumors: lesion conspicuity and extent. Radiology 179, 247-252.

43. Vormoor B, Knizia HK, Batey MA, Almeida GS, Wilson I, Dildey P, Sharma A, et al. (2014) Development of a preclinical orthotopic xenograft model of Ewing sarcoma and other human malignant bone disease using advanced in vivo imaging. PLoS One 9(1), e85128.

44. Welker JA, Henshaw RM, Jelinek J, Shmookler BM, Malawer MM (2000) The percutaneous needle biopsy is safe and recommended in the diagnosis of musculoskeletal masses. Cancer 89, 2677-2686.
45. Hau A, Kim I, Kattapuram S, Hornicek FJ, Rosenberg AE, Gebhardt MC, Mankin HJ (2002) Accuracy of CT-guided biopsies in 359 patients with musculoskeletal lesions. Skeletal Radiol 31, 349-353.

46. Skrzynski MC, Biermann JS, Montag A, Simon MA (1996) Diagnostic accuracy and charge-savings of outpatient core needle biopsy compared with open biopsy of musculoskeletal tumors. J Bone Joint Surg Am 78-A, 644-649.

47. Unni Krishnan K, Dahlin DC (1989) Osteosarcoma: pathology and classification. Semin Roentgenol 24, 143-152.

48. Mitsuyoshi G, Naito N, Kawai A, Kunisada T, Yoshida A, Yanai H, Dendo S, Yoshino T, Kanazawa S, Ozaki T (2006) Accurate diagnosis of musculoskeletal lesions by core needle biopsy. J Surg Oncol 94, 21-27.

49. Yi JH, Wang D, Li ZY, Hu J, Niu XF, Liu XL (2014) $\mathrm{C}$-Reactive protein as a prognostic factor for human osteosarcoma: A meta-analysis and literature review. PLoS One 9, 1-7.

50. Funovics PT, Edelhauser G, Funovics MA, Laux C, Berzaczy D, Kubista B, Kotz RI, Dominkus M (2011) Pre-operative serum $\mathrm{C}$-reactive protein as independent prognostic factor for survival but not infection in patients with high-grade osteosarcoma. Int Orthop 35, 1529-1536.

51. Hong AM, Millington S, Ahern V, Mccowage G, Boyle R, Tattersall M, Haydu L, Stalley PD (2013) Limb preservation surgery with extracorporeal irradiation in the management of malignant bone tumor: the oncological outcomes of 101 patients. Ann Oncol 24, 2676-2680.

52. O'Kane GM, Cadoo KA, Walsh EM, Emerson R, Dervan P, O'Keane C, Hurson B, et al. (2015) Perioperative chemotherapy in the treatment of osteosarcoma: a 26-year single institution review. Clin Sarcoma Res 5, 1-8.

53. Betz M, Dumont CE, Fuchs B, Ulrich Exner G (2012) Physeal distraction for joint preservation in malignant metaphyseal bone tumors in children. Clin Orthop Relat Res 470, 1749-1754.

54. Bacci G, Ferrari S, Longhi A, Forni C, Bertoni F, Fabbri N, Zavatta M, Versari M (2001) Neoadjuvant chemotherapy for high grade osteosarcoma of the extremities: long-term results for patients treated according to the Rizzoli IOR/OS-3b protocol. J Chemother 13, 93-99.

55. Ferrari S, Bertoni F, Mercuri M, Picci P, Giacomini S, Longhi A, Bacci G (2001) Predictive factors of disease-free survival for nonmetastatic osteosarcoma of the extremity: an analysis of 300 patients treated at the Rizzoli Institute. Ann Oncol 12, 1145-1150.

56. Ferrari S, Palmerini E, Staals EL, Mercuri M, Franco B, Picci P, Bacci G (2009) The treatment of nonmetastatic high grade osteosarcoma of the extremity: review of the Italian Rizzoli experience. Impact on the future. Cancer Treat Res 152, 275-287.

57. Malawer MM, McHale KA (1982) Limb-sparing surgery for high-grade malignant tumors of the proximal tibia, surgical technique and a method of extensor mechanism reconstruction. Clin Orthop Relat Res 239, 231-248.

58. Watts HG (1980) Introduction to resection of musculoskeletal sarcomas. Clin Orthop Relat Res 153, 31-38.

59. Wong KC, Kumta SM, Chiu KH, Antonio GE, Unwin P, Leung KS (2007) Precision tumour resection and reconstruction using image-guided computer navigation. J Bone Joint Surg Br 89, 943-947.

60. Krettek C, Geerling J, Bastian L, Citak M, Rücker F, Kendoff D, Hüfner T (2004) Computer aided tumor resection in the pelvis. Injury $35,79-83$. 
61. Khan FA, Lipman JD, Pearle AD, Boland PJ, Healey JH (2013) Custom jigs improve accuracy of wide resection of bone tumors. Clin Orthop Relat Res 471, 2007-2016.

62. Finn HA, Simon MA (1991) Limb-salvage surgery in the treatment of osteosarcoma in skeletally immature individuals. Clin Orthop Relat Res 262, 108-118.

63. Cañadell J, Forriol F, Cara JA (1994) Removal of metaphyseal bone tumours with preservation of the epiphysis. Physeal distraction before excision. J Bone Joint Surg Br 76, 127-132.

64. Kapoor S, Tiwari A, Kapoor S (2008) Primary tumours and tumorous lesions of clavicle. Int Orthop 32, 829-834.

65. Hillman A, Hoffmann C, Gosheger G, Krakau H, Winkelmann W (1999) Malignant tumor of the distal part of the femur or the proximal part of the tibia: endoprosthetic replacement or rotationplasty. J Bone Joint Surg Am 81, 462-468.

66. Schwartz AJ, Kabo JM, Eilber FC, Eilber FR, Eckardt JJ (2010) Cemented distal femoral endoprostheses for musculoskeletal tumor, improved survival of modular versus custom implants. Clin Orthop Relat Res 468, 2198-2210.

67. Gosheger G, Goetze C, Hardes J, Joosten U, Winkelmann W, von Eiff C (2008) The influence of the alloy of megaprostheses on infection rate. J Arthroplasty 23, 9-12.

68. Tsuchiya H, Nishida H, Srisawat P, Shirai T, Hayashi K, Takeuchi A, Yamamoto N, Tomia K (2010) Pedicle frozen autograft reconstruction in malignant bone tumors. J Orthop Sci $15,340-349$

69. Ahlmann ER, Menendez LR, Kermani C, Gotha H (2003) Survivorship and clinical outcome of modular endoprosthetic reconstruction for neoplastic disease of the lower limb. J Bone Joint Surg Br 88-B, 790-795.

70. Donati D, Di Liddo M, Zavatta M, Manfrini M, Bacci G, Picci P, Capanna R, Mercuri M (2000) Massive bone allograft reconstruction in high-grade osteosarcoma. Clin Orthop Relat Res 377, 186-194.

71. Gebhardt MC, Flugstad DI, Springfield DS, Mankin HJ (1991) The use of bone allografts for limb salvage in high-grade extremity osteosarcoma. Clin Orthop Relat Res 270, 181-196.

72. Gitelis S, Rasecki P (1991) Allograft prosthetic composite arthroplasty for osteosarcoma and other aggressive bone tumors. Clin Orthop Relat Res 270, 197-201.

73. Shalaby S, Shalaby H, Bassiony A (2006) Limb salvage for osteosarcoma of the distal tibia with resection arthrodesis, autogenous fibular graft and Ilizarov external fixator. J Bone Joint Surg Br 88-B, 1642-1646.

74. Sugiura H, Nishida Y, Nakashima H, Yamada Y, Tsukushi S, Yamada K (2012). Evaluation of long-term outcomes of pasteurized autografts in limb salvage surgeries for bone and soft tissue sarcomas. Arch Orthop Trauma Surg 132, 1685-1695.

75. Grimer RJ, Taminiau AM, Cannon SR (2002) Surgical outcomes in osteosarcoma. J Bone Joint Surg Br 84, 395-400.
76. Lang NW, Hobusch GM, Funovics PT, Windhager R, Hofstaetter JG (2015) What sports activity levels are achieved in patients with modular tumor endoprostheses of osteosarcoma about the knee? Clin Orthop Relat Res 473, $847-854$.

77. Stevenson J, Tsagkozis P, Grimer R (2016) Functional and quality of life outcomes in bone sarcoma following amputation, rotationplasty or limb-salvage. Expert Review of Quality of Life in Cancer Care 1, 303-312.

78. Salzer M, Knahr K, Kotz R, Kristen H (1981) Treatment of osteosarcomata of the distal femur by rotation-plasty. Arch Orthop Trauma Surg 99, 131-136.

79. Han G, Bi WZ, Xu M, Jia JP, Wang Y (2016) Amputation versus limb-salvage surgery in patients with osteosarcoma: a meta-analysis. World J Surg 40, 2016-2027.

80. Branemark R, Berlin O, Hagberg K, Bergh P, Gunterberg B, Rydevik B (2014) A novel osseointegrated percutaneous prosthetic system for the treatment of patients with transfemoral amputation: A prospective study of 51 patients. Bone Joint J 96-B, 106-113.

81. Harris JD, Thai QT, Thomas JS, Mayerson JL (2013) Exceptional functional recovery and return to high-impact sports after van nes rotationplasty. Orthopedics 36, 126-131.

82. Kotz R, Salzer M (1982) Rotation-plasty for childhood osteosarcoma of the distal part of the femur. J Bone Joint Surg Am 64, 959-969.

83. Gradl G, Postl LK, Lenze U, Stolberg-stolberg J, Pohlig F, Rechl H, Schmitt-sody M, Eisenhart-rothe RV, Kirchhoff C (2015) Long-term functional outcome and quality of life following rotationplasty for treatment of malignant tumors. BMC Musculoskelet Disord 16, 1-7.

84. Imran H, Enders F, Krailo M, Sim F, Okuno S, Hawkins D, Neglia J, et al. (2009) Effect of time to resumption of chemotherapy after definitive surgery on prognosis for nonmetastatic osteosarcoma. J Bone Joint Surg Am 91, 604-612.

85. Jones KB, Ferguson PC, Lam B, Biau DJ, Hopyan S, Deheshi B, Griffin AM, White LM, Wunder JS (2012) Effects of neoadjuvant chemotherapy on image-directed planning of surgical resection for distal femoral osteosarcoma. J Bone Joint Surg Am 94, 1399-1405.

86. Mason NJ, Gnanandarajah JS, Engiles JB, Gray F, Laughlin D, Gaurnier-Hausser A, Wallecha A, Huebner M, Paterson Y (2016) Immunotherapy with a HER2-targeting Listeria induces HER2-specific immunity and demonstrates potential therapeutic effects in a phase I trial in canine osteosarcoma. Clin Cancer Res 22, 4380-4390.

87. Messerschmitt PJ, Rettew AN, Brookover RE, Garcia RM, Getty PJ, Greenfield EM (2008) Specific tyrosine kinase inhibitors regulate human osteosarcoma cells in vitro. Clin Orthop Relat Res 466, 2168-2175.

Cite this article as: Misaghi A, Goldin A, Awad M \& Kulidjian AA (2018) Osteosarcoma: a comprehensive review. SICOT-J, 4, 12 